\title{
Influence of Head-Neck Rotation on Elbow Flexor and Extensor Muscle Activity and Strength in Normal Adults
}

\author{
Seung-Min Nam', Seong-Gil Kim² \\ 'Department of Physical Therapy, Daegu University, Daegu, Republic of Korea; ${ }^{2}$ Department of Physical Therapy, Sun Moon University, Asan, \\ Republic of Korea
}

Purpose: This study examined the effects of the directions of neck rotation position on the muscle activity and strength of the elbow flexor and extensor muscle.

Methods: Forty-one healthy adults participated in this study. The subjects were asked to their elbow $90^{\circ}$ flexion in three different neck rotations (neutral, ipsilateral, and contralateral) in the sitting position. The muscle activities of the biceps and triceps brachii muscle were measured using surface electromyography. And the muscle strength of the elbow flexor was measured using dynamometer. One way repeated measures ANOVA was used to compare the muscle activity and strength of the elbow flexor and extensor depending on the different neck turning directions.

Results: There were significant differences between contralateral neck rotation and ipsilateral neck rotation, contralateral neck rotation and neutral position. But, there was no significant difference in the triceps brachii muscle activity in comparison with the neck rotation. There were significant differences between contralateral neck rotation and ipsilateral neck rotation, contralateral neck rotation and neutral position.

Conclusion: To summarize this study, the elbow flexor and extensor muscle activity and strength was highest in the contralateral neck rotation position. In other words, it was possible to confirm the effect of Asymmetrical Tonic Neck Reflex in healthy adults whose primitive reflexes were inhibition, and head and neck positions should be considered during clinical evaluation and treatment.

Keywords: Head neck rotation, Biceps brachii, Triceps brachii, Muscle activity, Muscle strength

\section{INTRODUCTION}

Tonic Neck Reflex (TNR) refers to changes in limb muscle activity according to changes in head and neck positions. In relation to TNR, animal studies in which the cerebral cortex was removed showed that the muscle tone of the limbs varied depending on the position of the head. These reflexes are caused by the stimulation of proprioceptive receptors located in the neck. ${ }^{1}$ TNR is divided into Symmetrical Tonic Neck Reflex (STNR) and Asymmetrical Tonic Neck Reflex (ATNR). ATNR refers to the change in muscle tone of the limb according to head-neck rotation. In general, in ATNR when the head and neck are rotated, the extension and abduction of the limb occurs in the ipsilateral; the contralateral shows limb flexion and adduction. In particular, this reflection is more pronounced in the upper extremities than in the lower extremities. ${ }^{2}$
Primitive reflexes, such as ATNR, gradually decrease as they are inhibited by reaction reflex and equilibrium reaction. In general, ATNR has been reported to disappear at the five-month-old levels, and the appearance of this reflex after that has been reported as a sign of abnormal development. ${ }^{3}$ In addition, it has been reported that in normal adults head rotation does not affect elbow flexor or extensor strength, and ATNR does not affect normal adults. ${ }^{4}$ However, according to other previous studies, it has been reported that these reflexes may appear in normal adults even after ATNR is incorporated, and it was reported that this should be considered when setting up a treatment plan for the upper limbs. ${ }^{5}$ In addition, studies were conducted to determine whether ATNR affects the muscle tone and strength of the limbs even in patients with neurological injuries, such as stroke and traumatic brain injury. In general, ATNR occurs in patients with neurological impairment because motor control reverts to a state pri-

Copylight (C)2020 The Korean Society of Physical Therapy

This is an Open Access article distribute under the terms of the Creative Commons Attribution Non-commercial License (https:// creativecommons.org/license/by-nc/4.o.) which permits unrestricted non-commercial use, distribution, and reproduction in any medium, provided the original work is properly cited. 
or to integration. In other words, it has been reported that remapping of normal motor control is possible by using the change in muscle tone of the limbs according to head-neck rotation. ${ }^{6,7}$ However, according to other previous studies, it has been reported that the head-neck rotation of stroke patients did not change the activity and force of the upper limb muscles on the affected side. ${ }^{8}$

As such, studies on how head-neck rotation affects limb muscle activity in patients with nervous system damage, including normal adults with a loss of primitive reflex, have shown contradictory results. In addition, most previous studies were studies comparing ipsilateral rotation and neutral position, and subjective upper limbs muscle strength according to the head-neck rotation direction was measured. Since the effect of ATNR increases elbow flexor tone when head-neck ipsilateral rotation is performed, most of the previous studies compared ipsilateral rotation and neutral position. However, in order to accurately confirm the effect of ATNR, studies according to three positions of head-neck ipsilateral rotation, contralateral rotation, and neutral position are required. Therefore, the purpose of this study is to investigate how the head-neck ipsilateral rotation, contralateral rotation, and neutral position affect the flexor and extensor muscle activity and strength of the upper limbs in normal adults. This study also aims to provide evidence on whether head and neck positions should be considered during clinical evaluation and treatment.

\section{METHODS}

\section{Subjects}

This study was conducted on 41 university students (5 males and 36 females) enrolled in U University in Gyeongsangbuk-do; the age profile was $21.4 \pm 0.5$ (mean \pm standard deviation) years old, the height profile was $162.3 \pm 6.6 \mathrm{~cm}$, and the weight profile was $55.2 \pm 11.5 \mathrm{~kg}$ (Table 1). The criteria for selecting subjects were as follows: those who did not have any special diseases that could affect the study, had no visual or auditory damage, had no problems with the nervous system or vestibular organs. Before the experiment, all subjects were informed of the general purpose and procedure of this study, and they voluntarily agreed to participate in the experi-

Table 1. General characteristics of subjects

\begin{tabular}{lc}
\hline & Subject $(n=41)$ \\
\hline Gender (M/F) & $5 / 36$ \\
Age $(\mathrm{yr})$ & $21.4 \pm 0.5$ \\
Weight $(\mathrm{kg})$ & $55.2 \pm 11.5$ \\
Height $(\mathrm{cm})$ & $162.3 \pm 6.6$ \\
\hline
\end{tabular}

ment.

\section{Measurements}

1) Muscle strength

The Manual Muscle Test (MMT) was measured using a handheld dynamometer (Commander Muscle Tester, JTech, USA) to obtain a measurement of elbow flexor muscle strength. For the measurement posture, shoulder 90 degree abduction and elbow 90 degree flexion were performed. The subject tried to flex the elbow, and the measurer performed the MMT. The measurement was performed by one researcher, and the average value was used for a total of three time measurements. In order to avoid shake during the measurement, another person assisting the measurer held the arm in place. The dynamometer placement was proximal to the wrist joint on the radial surface of the forearm. ${ }^{9}$

\section{2) Muscle activity}

To check the muscle activity of the elbow flexor and extensor, a wireless system (FreeEMG 1000, BTS, Italy) was used, and the electrode was measured using $\mathrm{Ag}-\mathrm{Ag} / \mathrm{Cl}$. The electrode attachment sites were the muscle bellies of the biceps brachii and triceps brachii muscles. ${ }^{10}$ The EMG signals were collected at a signal acquisition rate of $1,000 \mathrm{~Hz}$ and then processed by full-wave rectification. Data was processed by band pass filtering at 30 $500 \mathrm{~Hz}$ using BTS EMG-Analyzer (BTS, Italy) software and notch filtering at $60 \mathrm{~Hz}$ to remove noise before storage. In order to standardize the measured values of each muscle, the measured raw data were converted into Root Mean Square (RMS) values; the average value of the three repeated measurements was obtained, and the muscle activity was analyzed. In addition, MVIC (Maximum Voluntary Isometric Contraction) was measured for each muscle, and muscle activity was normalized based on the value obtained. ${ }^{11}$

\section{Protocol}

Before conducting the experiment all subjects were asked to explain and understand the experiment method and procedure, and the researcher showed the demonstration and conducted the experiment. Subjects had their muscle strength measured using a dynamometer while performing elbow flexion in a sitting posture. During muscle strength measurement, EMG electrodes were attached to the biceps brachii and triceps brachii muscles, and the activity was measured. Measurements were made on the dominant hand. Also Muscle strength was measured in three postures based on the dominant hand: ipsilateral neck rotation, the neutral posi- 
tion, and contralateral neck rotation, respectively. During each posture measurement, if the subject complained of fatigue, sufficient rest was taken until they felt able to continue. In addition, in order to prevent muscle fatigue from affecting the measurement result, it was measured once per position per day, and the schedule was adjusted and measured by dividing it into different days. All measurements were made three times, and the measurement results were expressed as mean \pm standard deviation.

\section{Statistical analysis}

SPSS for Windows (version 22.0) was used for the data analysis of this study. Descriptive statistics were used to obtain general characteristics of the subject. In order to compare the difference in biceps brachii and triceps brachii muscle activity according to head-neck rotation and the muscle strength of the elbow flexor, the analysis was performed using repeated measurement ANOVA, and least significant difference (LSD) was used for post hoc analysis. Measurement results were expressed as mean \pm standard deviation. The statistical significance level was set to $\alpha=0.05$.

\section{RESULTS}

\section{Comparison of muscle activity according to neck rotation}

Contralateral neck rotation showed the highest biceps brachii muscle activity. There were significant differences between contralateral neck rotation and ipsilateral neck rotation, and between contralateral neck rotation and the neutral position $(\mathrm{p}<0.05)$. However, there was no significant difference in the triceps brachii muscle activity in comparison with the neck rotation $(\mathrm{p}>0.05)($ Table 2$)$.

\section{Comparison of elbow flexor strength according to neck rotation}

Contralateral neck rotation showed the highest elbow flexor strength. There were significant differences between contralateral neck rotation and ipsilateral neck rotation, and between contralateral neck rotation and the neutral position $(\mathrm{p}<0.05)($ Table 2$)$.

\section{DISCUSSION}

ATNR is the change in muscle tone of the limb according to head-neck rotation. Studies on how head-neck rotation affects limb muscle activity in patients with nervous system injuries, including normal adults with lost primitive reflex, have shown contradictory results. Therefore, the aim of the present study was to investigate the effect of head-neck rotation positions on the elbow flexor and extensor muscle activity and force in healthy young adults.

The results of the study indicated that the measurement of elbow flexor muscle activity in the contralateral neck rotation position yields the greatest activity. These findings are consistent with the findings that elbow flexor muscle tone increased when contralateral neck rotation was performed. ${ }^{12}$ ATNR has been reported to have reflexes in developing children and young adults. This suggested that head-neck rotation should be considered when improving the muscle strength of the upper extremities to utilize the influence of ATNR. ${ }^{4,13}$ It has also been reported that elbow muscle activity is affected by the position of the shoulder. ${ }^{14}$ Previous studies have reported that upper trapezius muscle activity is increased in the contralateral neck rotation position. ${ }^{15,16}$ That is, in this study, the muscles of the shoulder joint could be used when performing elbow flexion maximum isometric contraction; it is thought that the increase in upper trapezius muscle activity in the contralateral neck rotation position led to an increase in elbow flexor muscle activity in the contralateral neck rotation position.

On the other hand, there was no significant difference in elbow extensor muscle activity according to neck rotation position. These results are considered to be due to the measurement posture. According to previous studies, the antagonist muscle is reciprocal inhibition occurs when the agonist muscle contracts. ${ }^{17}$ In other words, in this study, since elbow extensor muscle activity was measured in a 90 degree flexion position of the elbow in the sitting posture, it is considered that there was no difference in elbow extensor muscle activity as measured with the antagonist muscle.

The results of the present study indicate that the measurement of elbow

Table 2. Comparison of muscle activity and strength depending on neck rotation directions

\begin{tabular}{|c|c|c|c|c|c|}
\hline \multirow{2}{*}{ Variable } & \multicolumn{3}{|c|}{ Neck rotating directions } & \multirow{2}{*}{$\mathrm{F}$} & \multirow{2}{*}{$p$} \\
\hline & Ipsilateral & Neutral & Contralateral & & \\
\hline Biceps M (\%MVIC) & $73.82 \pm 18.53$ & $78.44 \pm 16.04$ & $78.96 \pm 16.21^{\star+}$ & 7.35 & 0.002 \\
\hline Triceps M (\%MVIC) & $24.83 \pm 20.80$ & $27.01 \pm 24.28$ & $27.05 \pm 24.08$ & 1.71 & 0.197 \\
\hline Elbow flexor strength (lbs) & $28.84 \pm 8.83$ & $30.85 \pm 10.75$ & $31.39 \pm 11.88^{\star+}$ & 7.54 & 0.002 \\
\hline
\end{tabular}

Mean \pm SD: Mean \pm Standard Deviation, Biceps m: Biceps brachii muscle activity, Triceps m: Triceps brachii muscle activity

${ }^{*} \mathrm{p}$ <0.05: significant difference between neutral and contralateral neck rotation, ${ }^{\dagger} p<0.05$ : significant difference between ipsilateral and contralateral neck rotation. 
flexor muscle strength in the contralateral neck rotation position yields the greatest strength. These results are consistent with previous studies, which showed that the highest hand grip strength was measured at the contralateral neck rotation position as a result of evaluating hand grip strength according to the head-neck rotation position. ${ }^{18}$ The results showed slightly better flexor strength measurement in the head-neck rotation position when compared to the neutral head-neck position. However, other previous studies did not find any effect of head-neck rotation on the strength of the elbow flexor and extensor in healthy adults. ${ }^{19}$ This is considered to be a result of the gender of the study subjects. Previous studies concluded that the influence of the tonic neck reflex may be elicited more easily in female volunteers than in males, which is primarily due to the reduced limb strength in females. ${ }^{20}$ The proportion of women among the 41 subjects in this study was about $87 \%$, and it is thought that there was a difference in elbow flexor muscle strength according to the neck rotation position.

To summarize this study, the elbow flexor and extensor muscle activity and strength was found to be highest in the contralateral neck rotation position. In other words, it was possible to confirm the effect of Asymmetrical Tonic Neck Reflex in healthy adults whose primitive reflexes were inhibition. In addition, the position of the neck can affect the muscle strength and muscle activity of the upper extremity muscles according to the change of the muscle chain by muscle synergy. Therefore, when applying an intervention for functional recovery of the upper extremity, the position of the neck should be considered. This study was limited to young adults only. Therefore, a similar study with elderly patients or patients with central nervous system damage is warranted. And the female subjects of the study had a high sex ratio. In future studies, it is necessary to adjust the sex ratio. In addition, further study is needed to assess the elbow flexor and extensor muscle activity and strength in different neck positions in patients with neck pain and upper limb dysfunction.

\section{REFERENCES}

1. Bruijn SM, Massaad F, Maclellan MJ et al. Are effects of the symmetric and asymmetric tonic neck reflexes still visible in healthy adults? Neurosci Lett. 2013;556:89-92.

2. Woollacott MH, Shumway Cook A, Nashner LM. Aging and posture control: changes in sensory organization and muscular coordination. Int J Aging Hum Dev. 1986;23(2):97-114.
3. Coryell J, Henderson A, Liederman J. Factors influencing the asymetrical tonic neck reflex in normal infants. Phys Occup Ther Pediatr. 1982;2(23):51-65.

4. Deutsch H, Kilani H, Moustafa E et al. Effect of head-neck position on elbow flexor muscle torque production. Phys Ther. 1987;67(4):517-21.

5. Bohannon RW, Andrews AW. Influence of head-neck rotation on static elbow flexion force of paretic side in patients with hemiparesis. Phys Ther. 1989;69(2):135-7.

6. Naghdi S, Ansari NN, Mansouri K et al. A neurophysiological and clinical study of Brunnstrom recovery stages in the upper limb following stroke. Brain Inj. 2010;24(11):1372-8.

7. Son SM, Hwang YT, Nam SH et al. Motor skill learning on the ipsi-lateral upper extremity to the damaged hemisphere in stroke patients. J Kor Phys Ther. 2019;31(4):212-5.

8. Andrews AW, Thomas MW, Bohannon RW. Normative values for isometric muscle force measurements obtained with hand-held dynamometers. Phys Ther. 1996;76(3):248-59.

9. Bohannon RW. Test-retest reliability of hand-held dynamometry during a single session of strength assessment. Phys Ther. 1986;66(2):206-9.

10. Hermens HJ, Freriks B, Disselhorst-Klug C et al. Development of recommendations for SEMG sensors and sensor placement procedures. J Electromyogr Kinesiol. 2000;10(5):361-74.

11. Yun SJ, Kim MH, Weon JH. Effect of shoulder position on scapular muscle activity during scapular protraction. J Kor Phys Ther. 2020; 32(3):157-62.

12. James TT, Nayak JS. Influence of the asymmetric tonic neck reflex on handgrip strength in healthy adults. J Indian Assoc Physiother. 2019;13 (2):90.

13. Lee JH, Yoo WG, Lee KS. Effects of head-neck rotation and kinesio taping of the flexor muscles on dominant-hand grip strength. J Phys Ther Sci. 2010;22(3):285-9.

14. Henkel M. Schrödinger invariance and strongly anisotropic critical systems. J Stat Phys. 1994;75(5-6):1023-61.

15. Shin YW, Kim CH, Han JT. Effect of neck rotating directions on the muscle activity of upper and lower trapezius during shoulder external rotation. J Kor Phys Ther. 2020;32(2):101-6.

16. Seo GJ, Park JW, Kwon Y. Comparison of the Electromyographic activity in the lower trapezius muscle according to four different types of exercises in healthy adults. J Kor Phys Ther. 2019;31(2):134-9.

17. Loh PY, Hayashi K, Nasir $\mathrm{N}$ et al. Changes in muscle activity in response to assistive force during isometric elbow flexion. J Mot Behav. 2020; 52(5):634-42.

18. Kumar NSS, Daniel CR, Hilda M et al. Grip strength: influence of headneck position in normal subjects. J Neuro Res. 2012;2(3):93-8.

19. Rex Wong YC, Cameron D, Bohannon RW. Elbow and hand muscle strength are not affected by head-neck position. Isokinet Exerc Sci. 1998;7(1):43-7.

20. Zafar H, Alghadir A, Anwer S. Effects of head-neck positions on the hand grip strength in healthy young adults: a cross-sectional study. Biomed Res Int. 2018;2018. 